\title{
An Aid Decision Tool for Implementing Wave Energy Devices on the Coast
}

\author{
André Martinez, Zied Ben Mustapha, Rose Campbell \\ Ecole d'Ingénieurs Généralistes en Génie des Systèmes Industriels (EIGSI), France
}

\begin{abstract}
A decision support tool for the selection of suitable areas is proposed in this paper for wave energy projects. It is based on a multi-criteria assessment methodology that considers physical, environmental and socioeconomic constraints. Criteria for site selection can include a large number of parameters, such as bathymetry and sea bottom. Additional factors (e.g. technological constraints, administrative and logistics conditions) can be added according to data availability; weight assigned for the different parameters can be easily adapted to suit the requirements of specific devices and installation procedures. The proposed method is applied to the French Atlantic Coast. Results show appropriate areas for the best locations for implementing a wave energy farm. French Brittany and the southern part of French Coast appear to be most suitable for wave energy devices deployment, not only from an energy point of view. The tool has been developed and tested over a large area. It can also be used in specific areas, with smaller scale, if geospatial data with good resolution are available.
\end{abstract}

\section{Introduction}

Given the current and projected global energy demand and the associated impact on the environment, marine energy conversion projects offer viable alternatives with their clean and renewable applications [1]. There are many projects and emerging technologies worldwide to convert the vastly unexploited ocean wave energy to electric power.

The location choice for a wave energy farm depends on the assessment of a number of criteria including the available power, site characteristics, environmental, economic and social factors related to the planned project [2]. To optimize energy extraction while limiting the environmental impacts and conflicting interests, it is essential to assess the relevant factors to the deployment of such a project. The available power and the site characteristics such as bathymetry, water depth and the geology of the seabed constitute the physical constraints of analysis, which are easier to assess quantitatively with modeling and measurements than the environmental, economic and social constraints. The connection of the device to the electric grid, its mooring and accessibility are generally identified as locationdependent cost factors.
In multi-criteria analysis, a significant amount of information needs to be implemented. This makes GIS tools particularly useful [3]. For the last decades, GIS applications have been successfully used to select most suitable areas for energy projects [4-8].

Geo-spatial multi-criteria analysis is a suitable technique to consider a wide variety of environmental and administrative factors (e.g. water depth, distance to shore, geology) and assign corresponding weights. A numerical result (suitability value) is returned as an output, in a given scale, for each location. The integration of multicriteria analysis with GIS allows the evaluation of various alternatives on the basis of multiple and conflicting criteria and objectives. There are many applications in the literature where GIS techniques have been combined with multi-criteria analysis [9]presents a GIS based multi-criteria analysis to identify the best sites for the construction of new wind farms. Nobre et al. [1] developed a geo-spatial multi-criteria analysis for wave energy converters deployment in Portugal. Carver [10] presents an application for the search of suitable places for the disposal of radioactive wastes in the UK. Zambon et al. [11] describes a GIS based multi-criteria decision analysis method for evaluating alternative places for the location of thermoelectric power plants in São Paulo state (Brazil).

This study aims at designing a site selection decision making tool for wave energy conversion (WEC) system deployment. This method allows choosing an appropriate location while minimizing technical constraints. However, since there is not a focus on a specific WEC system, final users must take into account the intrinsic characteristics of the WEC to be deployed (e.g. optimal wave energy and height, mooring depth) and, hence, estimate the impact each factor can have on the system so that weights are properly assigned.

In a multi-criteria analysis, the first step is to define the exclusion criteria. Those factors (e.g. marine protected areas, underwater cables) are used to define restriction areas, where the future wave farm cannot be implemented because of environmental, technical or administrative reasons. These areas are removed from the analysis zone. The second step is the definition of the weighting parameters (e.g. wave climate characteristics, distances to key areas such as coasts and power grid, bathymetry). Weights are then assigned to each factor according to its relative impact on the system 
implementation. For a specific system deployment, the final user should proceed to the evaluation of each factor importance and perform the weighting procedure together with expert opinions. All the weighted factors are finally overlaid to produce the site suitability GIS layer for potential wave farm deployment locations.

This paper is organized as follows: first, the used data are described and the methodology is summarized. Then, the criteria for site selection are listed and discussed. A step by step description of the methodology is afterwards described. The results are then presented and discussed. Finally, the concluding remarks and suggestions for future work are presented.

\section{Data and Methodology}

\subsection{Summarized Methodology}

In GIS, vector and raster are two different ways of representing spatial data. Vector consists of individual points. The points may be joined in a particular order to create lines, or joined into closed rings to create polygons. In addition to the geometric representation of features, vector data contains a set of attributes that characterize those features, increasing the analysis capabilities.

Raster data is made up of pixels (or cells), and each pixel has an associated value. The geographic location of each cell is implied by its position in the cell matrix. Due to the nature of the data storage technique data analysis is usually easy to program and quick to perform. The inherent nature of raster maps is ideally suited for mathematical modelling and quantitative analysis.

In this study, data acquisition was mainly performed in a vector structure, while most of the processing and analysis procedures were made using a raster structure. The geo-spatial multi-criteria analysis was developed on ArcGIS@ Desktop 10.2 geographic information system software, with the spatial analyst extension.

The developed methodology is summarized in Figure 1. The first step of the methodology is to select a study area and create the analysis mask according to a number of criteria. All the remaining steps of the analysis will be then performed within this masked area. Principle factors related to wave energy farms deployment are then identified. Once all criteria are defined, a common evaluation scale must be set. This procedure is known as "reclassification" in the GIS software used in the present study and performs a table lookup to set a common scale to all factors.

Afterwards, the identified factors are weighted according to their relative importance with respect to the future WEC deployment site. It is of particular importance that the weighting is performed by experts and verified by the project holder in order to adjust the decision making tool to a particular WEC system.

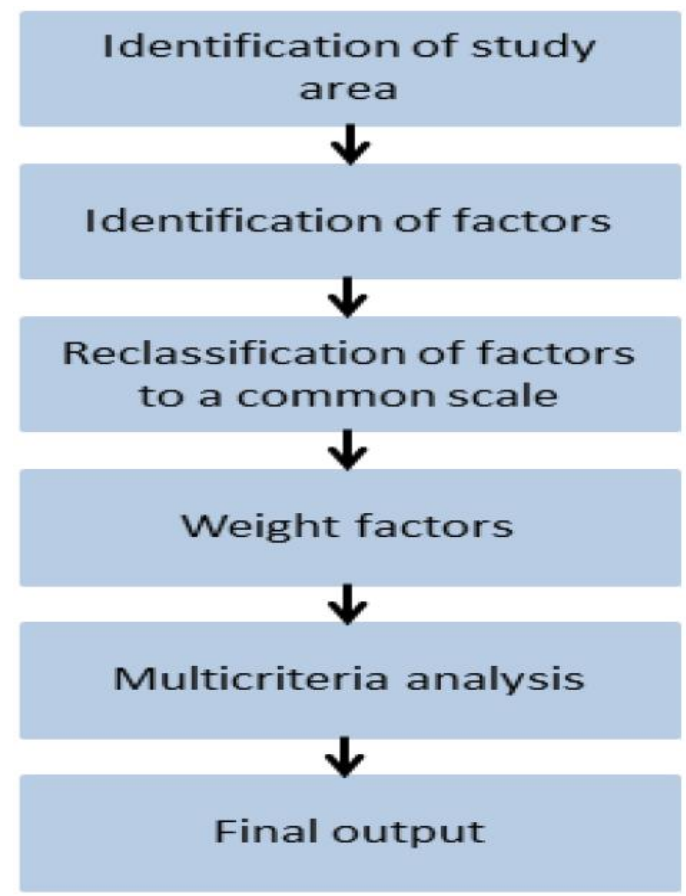

Figure 1. Summarized methodology

Geospatial analysis is then used to calculate the overall suitability matrix for implementing the WEC. This matrix will have values between 0 and 100, where the highest values represent the most suitable places to set the system. Decision makers will have at their disposal the necessary geographic information aid for analyzing and complementing the WEC implementation procedure.

\subsection{Data Acquisition}

Bathymetry data was acquired in raster format from the GEBCO database (General Bathymetric Chart of the Ocean (http://www.gebco.net/). The raster data have been then transformed to vector format using the "Contour" function available in ArcGIS@ Spatial Analyst extension in order to produce bathymetric contour lines (isobaths). Territorial water boundaries (Exclusive Economic Zone) were acquired from SHOM (naval hydrographic and oceanographic service). Sea bottom characteristics and underwater cables locations have been downloaded from the SEXTANT database (IFREMER).

Power grid lines and electric substation positions were downloaded from RTE (national electricity transmission network).

Shoreline position was downloaded from the EEA (European Environment Agency) database (http://www.eea.europa.eu/data-and-maps). Marine 
protected areas locations were acquired from the INPN database (national inventory of natural heritage, http://inpn.mnhn.fr/accueil/index).

Wave data (mean height and power) were obtained from the PREVIMER database (http://www.previmer.org/). Wave heights and directions are generated using the WAVEWATCHIII model on a regular grid basis, with a $200 \mathrm{~m}$ resolution. Data are generated every hour with a 6 days forecasting window.

\subsection{Criteria for site selection}

The methodology developed in this study has been applied to the French Atlantic area. This case was set as a methodology demonstration and does not apply to any specific WEC. Raster resolution was set to $200 \mathrm{~m}$. This means that the final suitability layer will present a score for every $200 \mathrm{~m}$ x $200 \mathrm{~m}$ square of the study area.

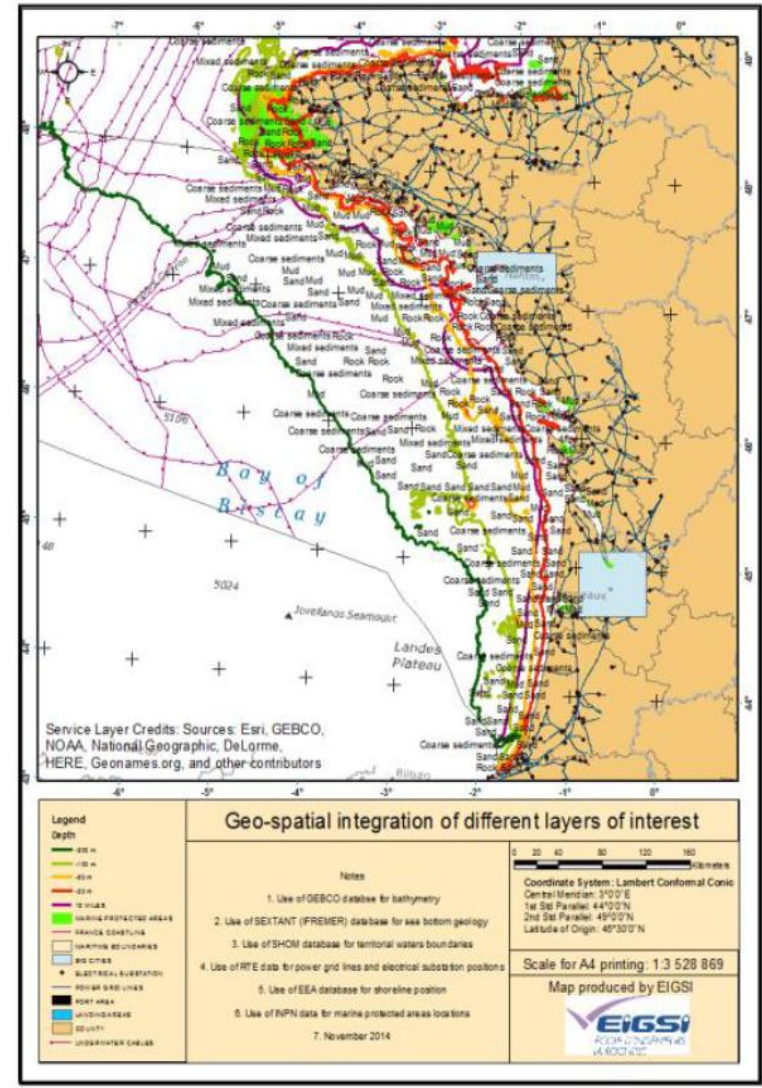

Figure 2. Geo-spatial integration of different layers

The first step in the development of a multicriteria decision making system is to define a general area of interest (i.e. study zone) for the analysis and the identification of features that may affect (or be affected by) the future wave energy farm location. These features include some locations where the system cannot be implemented. Therefore, they have to be removed from the overall area of interest, producing a mask for the analysis.

Considering most specifications for WEC, the following features locations were to be defined as exclusion areas: marine protected areas; five-hundred meters areas around underwater cables locations; harbor entrances and navigation channels; depths higher than $200 \mathrm{~m}$ and lower than $20 \mathrm{~m}$.

Features considered as selection criteria are: distance to coastline; distance to ports; distance to the electric network connection points; type of sea bottom; wave climatology (significant height and power).

The different data described above have been integrated into the GIS software, as shown in Figure 2.

\subsection{Creating the analysis mask}

The analysis mask (see Figure 3) was created by spatially removing areas related to:

- Underwater cables: the areas with such infrastructures should be avoided because cables can be broken due to actions such as anchoring. Therefore, a $500 \mathrm{~m}$ buffer around the cables was set as an exclusion area.

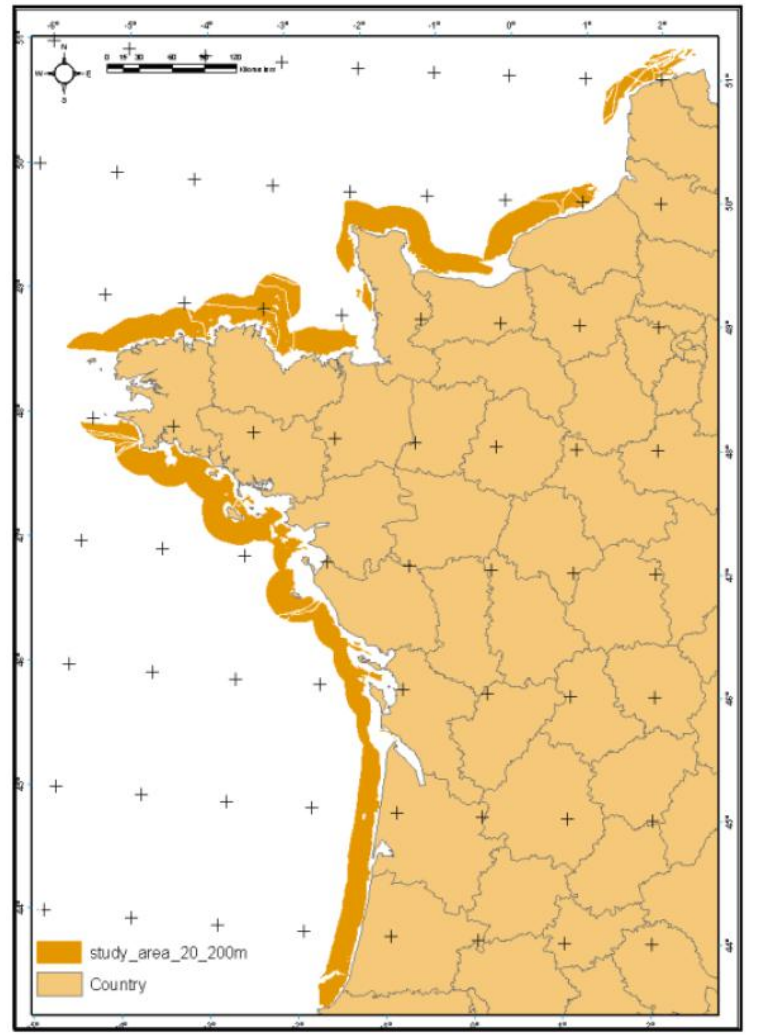

Figure 3. Final mask (interest area)

- Marine protected areas: these areas should not be included in the analysis because of the inherent legislations related to the protection of living organisms and natural habitats. 
- Ocean depths: bathymetry should be appropriate for the implementation of wave energy devices. For technical and economic reasons, for most offshore wave energy conversion systems, sea bottom must be deeper than $20 \mathrm{~m}$ and shallower than $200 \mathrm{~m}$. However, depth criteria should be adapted in order to be suitable to the specific WEC to be deployed.

\subsection{Creating and reclassifying weighted factors}

The ArcGIS@ Spatial Analyst extension has been used to generate and reclassify the selection criteria. The data have been extracted from GIS datasets gathering coastline position, sea bottom nature, ports and electric substations locations as well as mean significant wave height and power.

With such a variety of parameters, scales and measuring units, it was necessary to define a common comparable scale that would convert such units to its corresponding expected impact in the WEC location problem. A suitability scale of 1 to 5 was used. For a given criterion, the best condition for implementing the WEC was rated 1 and the worst condition was rated 5

The Euclidean distance tool, measures the distance from a GIS layer feature to each raster cell, was applied to the coastline, ports and electric substations. . Using this tool, 3 raster maps (Figures 4-6) were obtained that represent the distance to the referred themes (the shortest distance, the better).

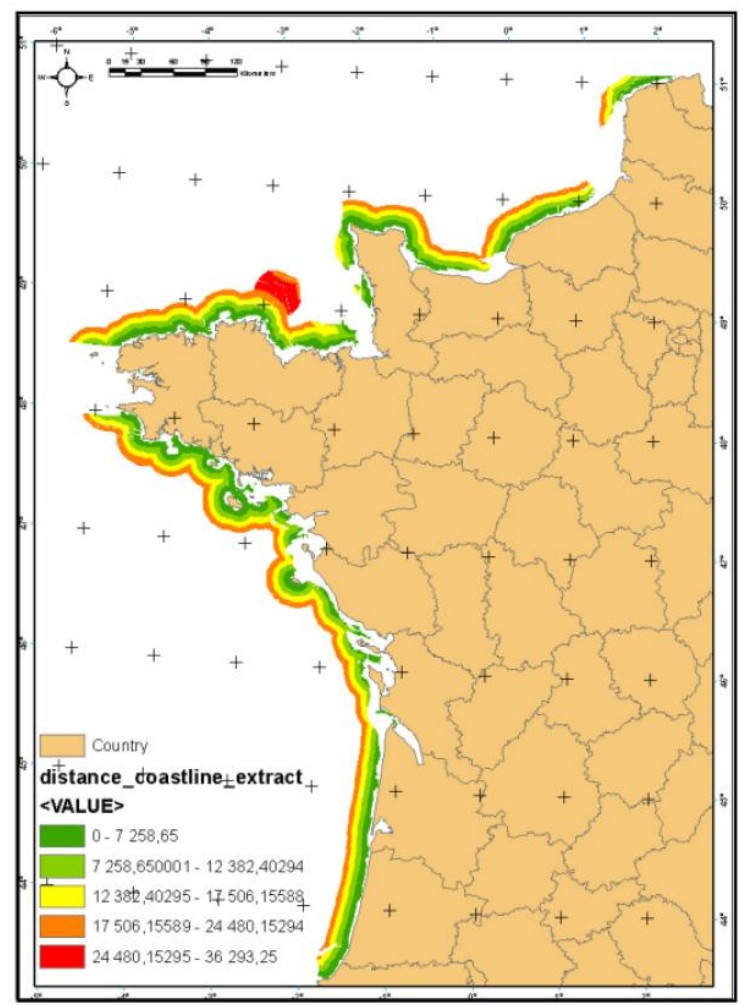

Figure 4. Distance to coastline (kms)

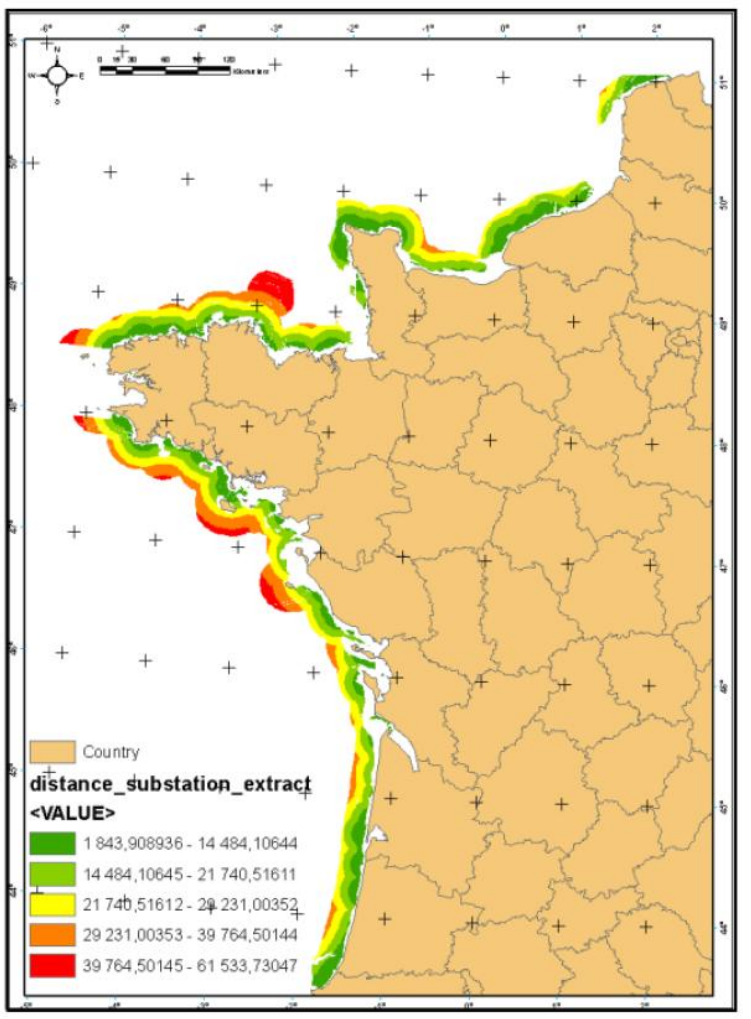

Figure 5. Distance to electric substation (kms)

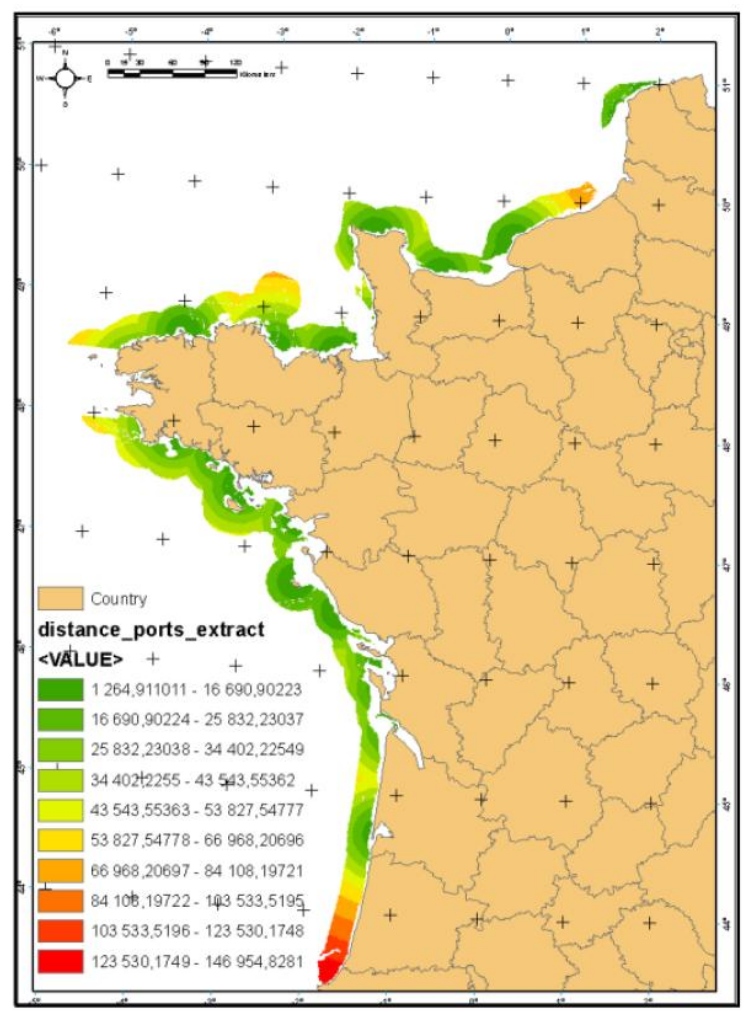

Figure 6. Distance to ports (kms) 


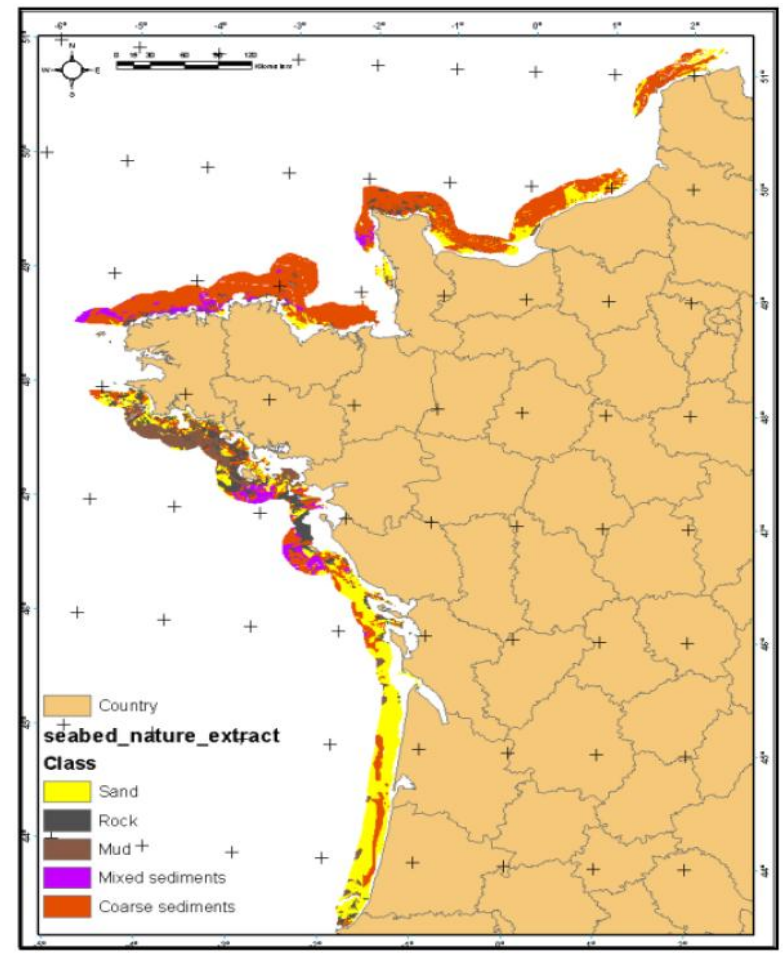

Figure 7. Sea bottom characteristics

For the sea bottom characteristics features, better location ratings have been assigned to sandy bottomed areas (for the facility of anchoring operations) while lower location ratings have been assigned to hard sea bottomed areas (rocky or coarse sediments). Middle ratings have been assigned to muddy and mixed sediments locations (see Figure 7).

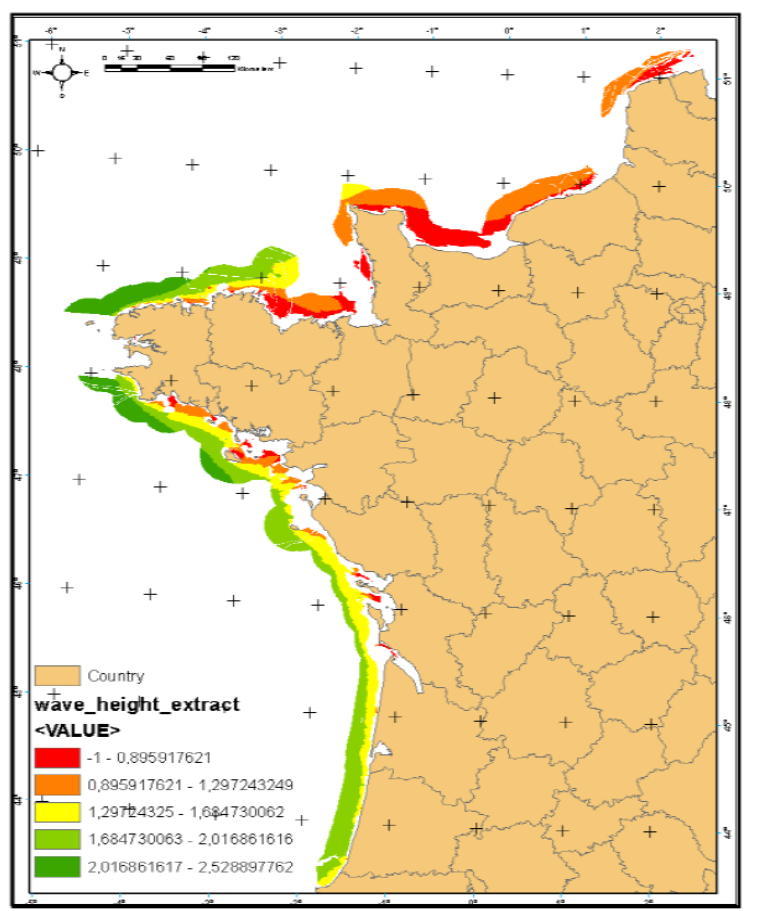

Figure 8. Mean wave height (in $\mathrm{m}$ )
An IDW (Inverse Distance Weighted) interpolation was used to obtain raster datasets of wave climatology data (mean height and power). The calculated raster maps are presented in Figures 8 and 9.

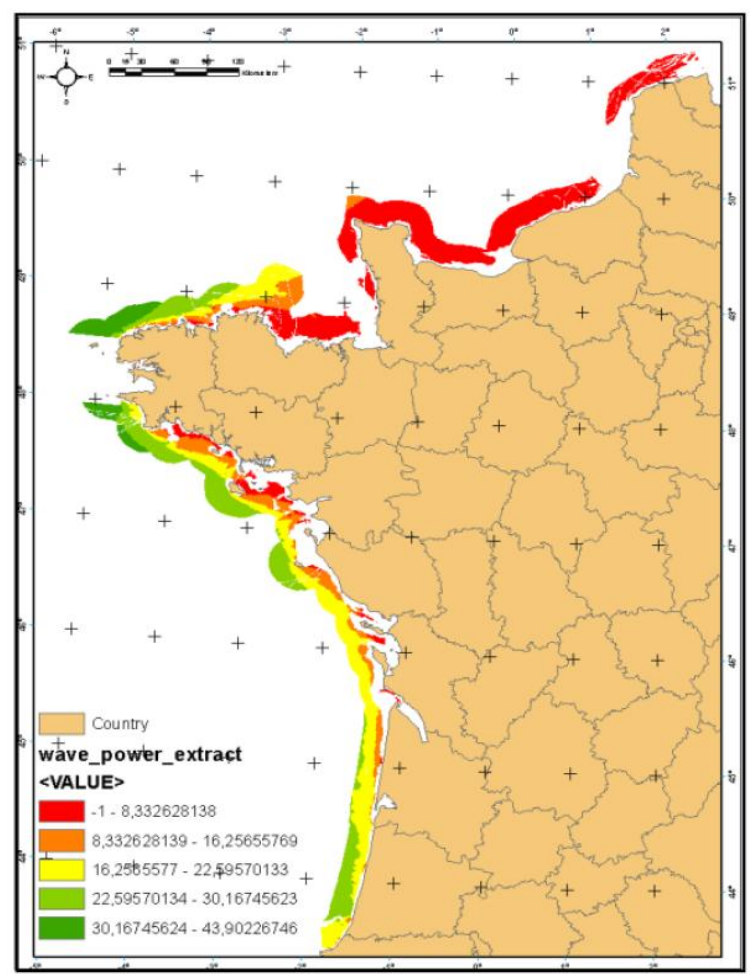

Figure 9. Reclassification of mean waves power $(\mathrm{Kw} / \mathrm{m})$

The next step is also very important to the multicriteria analysis procedure: setting the weight of each reclassified factor. In this study, different weights configurations have been tested.

\subsection{Weighting factors}

Now that all the criteria are reclassified to a common scale, it is necessary to determine each theme's relative impact in the location selection problem. In this phase, weights were assigned to factors according to that impact. Based on the different data layers and their assigned weights, a suitability raster layer is produced as a final output using the weighted overlay procedure (ArcGIS $\odot$ Spatial Analyst extension). The Weighted Overlay tool applies one of the most used approaches for overlay analysis to solve multi-criteria problems such as site selection and suitability models. This operation represents the last step of the multi-criteria decision making system before creating the output raster (suitability of each location for future device deployment). Weighted overlay is an intersection of standardized and differently weighted layers in a suitability analysis. The weights quantify the relative importance of the suitability criteria considered. The 
algorithm multiplies all grid cells of a layer by their weights. Then, the weighted layers are added together. In the resulting suitability layer, the suitable cells have high values while the not suitable cells have low values.

1) Select an evaluation scale: Values at one end of the scale represent one extreme of suitability (or other criterion); values at the other end represent the other extreme. It is important to select an evaluation scale that matches the scale used when reclassifying. For instance, if raster maps were reclassified using a scale of 1 to 5 (as it was done in this study), an evaluation scale of 1 to 5 by an increment of 1 or 0 to 100 by an increment of 20 should be entered for the evaluation scale in the weighted overlay. In this study, the latter evaluation scale has been chosen.

2) Add raster maps: previously reclassified factors are added one by one to the weighted overlay table.

3) Set scale values: the scale values for each input raster in the analysis are assigned values from the evaluation scale. This makes it possible to perform arithmetic operations on raster maps that originally held dissimilar types of values. It is possible to change the default scale values according to importance or suitability.

4) Assign weights to input raster maps: Each input raster can be weighted, or assigned a percentage influence, based on its importance. The total influence for all raster maps must equal 100 percent.

5) Run the weighted overlay tool: the cell values of each input raster are multiplied by the raster's weight (or percent influence). The resulting cell values are added to produce the final output raster.

In order to assess the sensitivity of the multicriteria analysis tool to the chosen weights, three configurations have been tested. Table 1 presents the weights that were assigned to the different factors. In the first test, $60 \%$ of the weights have been assigned to the energy factors (wave height and power). In the second test, $50 \%$ of weights have been allocated to waves, giving more importance to the sea bottom characteristics when compared to the first test. In the third configuration, $80 \%$ of the weights have been assigned to the wave parameters.

Table 1. Weights assigned to the different themes for the two tested configurations

\begin{tabular}{|c|c|c|c|}
\hline Criteria & $\begin{array}{c}\text { Weights } \\
\left(1^{\text {st }} \text { test }\right) \\
(\%)\end{array}$ & $\begin{array}{c}\text { Weights } \\
\left(2^{\text {nd }} \text { test }\right) \\
(\%)\end{array}$ & $\begin{array}{c}\text { Weights } \\
\left(3^{\text {rd }} \text { test }\right) \\
(\%)\end{array}$ \\
\hline $\begin{array}{c}\text { Sea bottom } \\
\text { characteristics }\end{array}$ & 10 & 20 & 5 \\
\hline Distance to shore & 10 & 10 & 5 \\
\hline Distance to ports & 10 & 10 & 5 \\
\hline $\begin{array}{c}\text { Distance to } \\
\text { substation }\end{array}$ & 10 & 10 & 5 \\
\hline Waves height & 30 & 25 & 40 \\
\hline Waves power & 30 & 25 & 40 \\
\hline
\end{tabular}

\subsection{Using a geo-processing model to automate the different tasks}

The multi-criteria analysis described in this study needs some testing procedures in order to assess the impact of each selection criterion on the final result [2].

Manually processing and generating all the data layers can be a very repetitive and error prone procedure. Therefore, the implementation of a routine using a geo-processing model makes the system testing and calibration efficient. ModelBuilder is an application used to create, edit, and manage models. Models are workflows that string together sequences of geo-processing tools, feeding the output of one tool into another tool as input. ModelBuilder can also be thought of as a visual programming language for building workflows. Any change in the multi-criteria tool parameters (e.g. reclassification values, assigned weights) can be directly made in the geo-processing model environment and running the whole procedure is automatically performed in a few minutes.

For each of the three steps described above (Sections 2.5-2.7), a processing model has been created. The inputs of the model (represented in blue, Figure 10) are data layers gathering the main criteria for site selection described in section 2.3. The tools, such as buffering and intersection or union operations are represented by the yellow boxes. Finally, the output layers or intermediate outputs are represented by the green boxes.

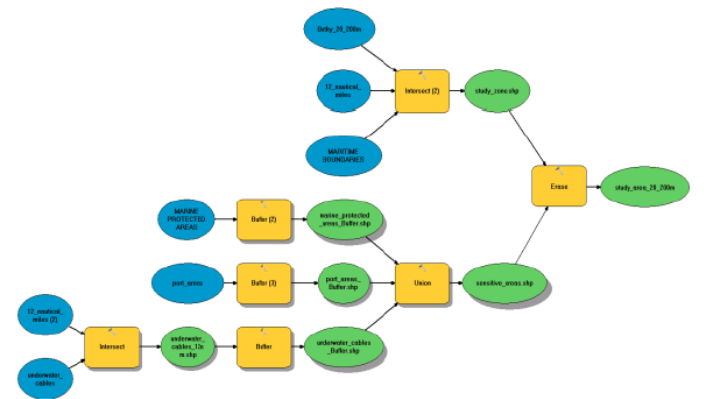

Figure 10. Creation of the analysis mask processing model

The first processing model (see Figure 10) creates the analysis mask (i.e. study area_20_200m in Figure 10). The sensitive areas (marine protected areas, ports and underwater cables) are spatially removed using the "Erase" tool.

The second processing model (see Figure 11) calculates the Euclidean distance separating each raster cell of the analysis mask from the coastline, ports and electric substation layers. Then, all the data layers are extracted within the analysis mask using the "extract by mask" tool. 


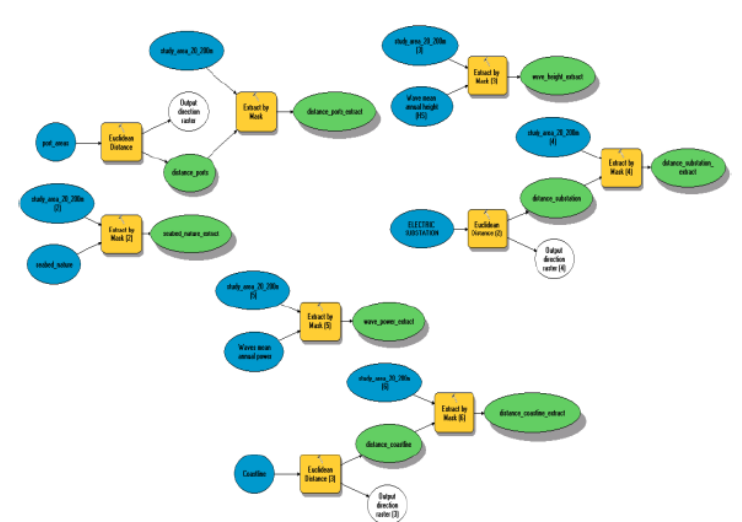

Figure 11. Data extraction in the analysis mask processing model

The third processing model (see Figure 12) reclassifies the input factors in order to set them into the same scale (see section 2.6). The "reclassify" tool allows setting a score for each factor on a scale going from 1 to 5,1 being the best score (e.g. closest points from the coast, ports areas, sandy sea bottom) and 5 the worst (e.g. most distant points from the coast, ports areas, rocky sea bottom). The reclassification procedure gives six intermediate variables (e.g. reclassified_wave_power) each having the same scale. These variables will be used as inputs for the weighted overlay procedure.

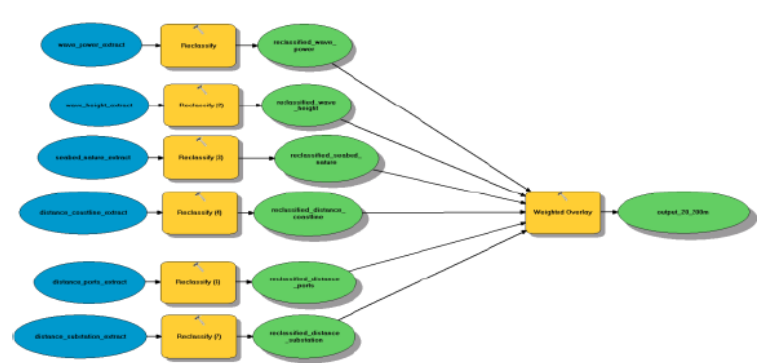

Figure 12. Reclassification and weighted overlay processing model

Once all the parameters described above are set, the tool runs automatically within the third processing model (see Figure 12). The tool parameters can be changed if needed.

\section{Results}

The resulting suitability layers are presented in Figures 13-15. The overall results (for the three tests) show that the most suitable places are located in the Atlantic coasts in the western part of France as well as in French Brittany, where the highest wave energy resources are located. On the other hand, less suitable areas are located in the English Channel, where wave resources are weak.

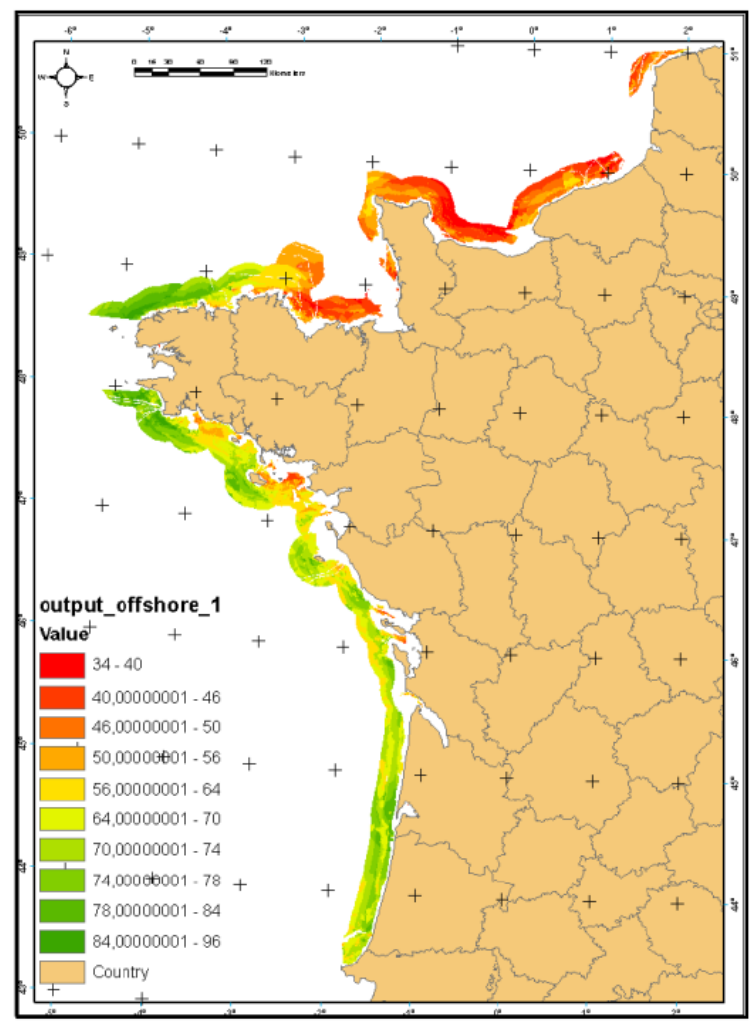

Figure 13. Final output (1st test)

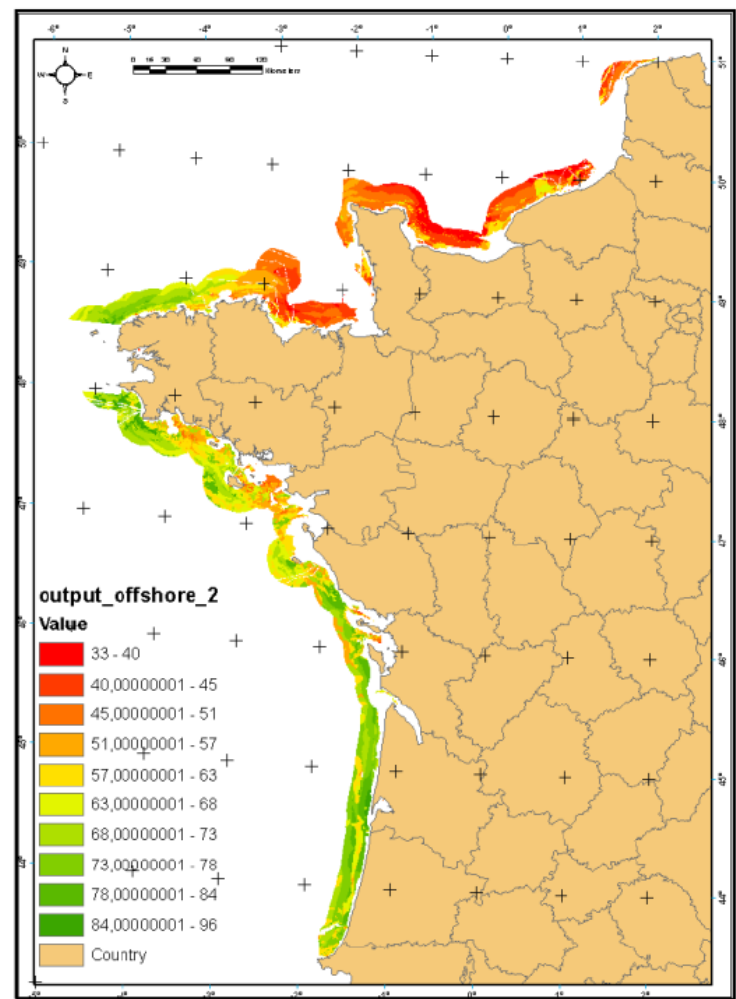

Figure 14. Final output (2nd test) 


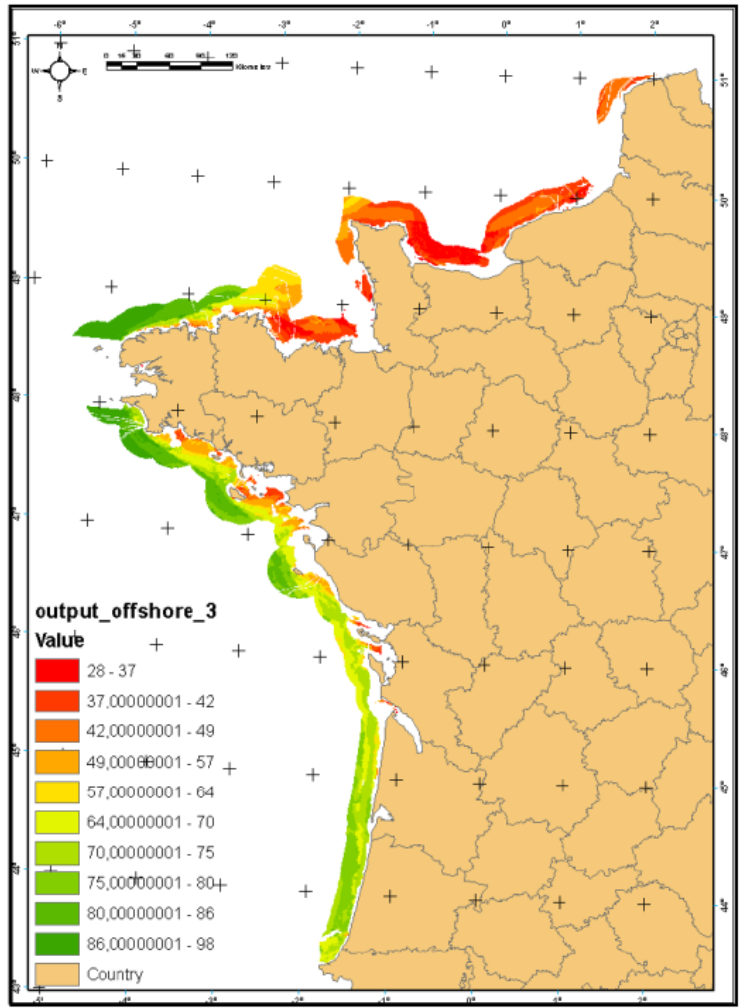

Figure 15. Final output (3rd test)

Again, it is of particular importance to highlight that the results obtained in this study are not focused on a particular wave system. The most suitable sites represent the locations with the highest wave energy resources and where the technical constraints are minimized (e.g. water depth, distance to shore, etc.). Final users and decision makers will have to adapt the tool according to their own system's characteristics.

In the wave shadowed areas, the most suitable sites for the wave energy device deployment are relatively far from the coast. For instance, in some areas (e.g. Vendée and Charente-Maritime regions), the closest suitable zones with the highest score are at approximately $50 \mathrm{~km}$ from the coast. This is due to the coastline orientation compared to the incident wave direction as well as the sea bottom slope (bathymetric constraint as depth has to be higher than $20 \mathrm{~m}$ ). These locations are, by nature, not efficient for wave energy conversion because the combination of the most frequent wave directions and shore location creates shadow zones. Moreover, for economic, technical and operational reasons (e.g. connexion to the power grid, operation and maintenance), the device has to be as close as possible from the coast. Therefore, those areas are to be avoided for a wave energy conversion system deployment [2].

On the other hand, locations with higher sea bottom slopes (i.e. higher depths closer the coast), facing directly incident waves have to be particularly considered by projects leaders. These areas are mainly located around French Brittany as well as in the southern part of the French Atlantic coasts.

The comparison between the three weights configurations shows that the multi-criteria analysis tool markedly responds to the difference between the weights assigned to the different parameters. For the first and second configurations (respectively 60\% and $50 \%$ of the weights assigned to the wave parameters), Figures 10 and 11 shows patchy structures in the raster representing the final results. These heterogeneities are due to the influence of sea bed nature (see Figure 7), which has, respectively, 10 $\%$ and $20 \%$ of the assigned weights in the first and second tests. However, the third weight configuration (5\% of the weights for the seabed nature and $80 \%$ for wave parameters) generates smoother structures, mainly corresponding to the distribution of the wave energy resources, as shown in Figure 15. This comparison shows that the methodology is perfectly sensitive to weights parametrization. However, projects leaders should adapt the input parameters and the weights configuration according to their own system characteristics.

Areas with the highest potential are in agreement with the chosen experimentation sites for wave energy prototype devices deployment in France (e.g. SEM-REV experimentation offshore Le Croisic or the test site in the Audierne bay in French Brittany).

Other information that was not available for this study should also be taken into account when creating the analysis mask in order to avoid conflicting interests, such as, the position of military exercise areas, wrecks, harbour approaches, fishing and aquaculture areas. Collecting in situ data through automatic measurements or oceanographic campaigns is essential prior to any project development. Those include bathymetric surveys, wave characteristics measurements as well as fauna and flora inventories in the most suitable area identified by the multi-criteria analysis.

\section{Perspective use of the multi-criteria analysis tool: application for onshore wave projects deployment}

The multi-criteria analysis tool described above has been developed with an emphasis on offshore areas (depths going from 20 to 200m). The systems that are targeted for such areas are WECs that have been developed to extract energy from the deeper waters offshore or nearshore. Examples of such systems are attenuator WECs (e.g. Pelamis Wave Power, Scotland), oscillating wave surge converters (e.g. Oyster wave energy converter by Aquamarine Power, Scotland) or rotating mass (e.g. SEM-REV prototype, France). 
Other types of WECs, designed for onshore installation, exist or are under development. Among such systems, we can cite the oscillating water column systems (e.g. Oceanlinx prototype, Australia) or overtopping devices (e.g. WaveDragon, Denmark). Such devices can be installed on existing infrastructures, such as breakwaters or sea walls. Several infrastructures in the Atlantic coast of France could be used to install such devices. The multicriteria analysis tool can be used to locate the most suitable areas to deploy a WEC on existing infrastructures.

The same methodology has been used by considering depths between 0 and $20 \mathrm{~m}$. The analysis mask processing model (see Figure 10) is therefore used by replacing the bathymetry data. Therefore, this analysis mask covers only the range of depths between 0 and 20m (see Figure 13).

The two next steps (see Figures 11 and 12) have been applied within the new analysis mask (Euclidean distance calculation, data extraction in the analysis mask and reclassification procedures). In this case, the application of the methodology concerns the onshore wave projects deployment, the distance to the coast and the sea bottom characteristics have not been taken into account in the weighted overlay procedure. The weights used are $80 \%$ for wave parameters, $20 \%$ for distance to electric substations and $20 \%$ for distance to port areas.

Finally, the output raster has been clipped to keep only the output raster cells that are the closest to the coastline in order to have the onshore suitability of a wave energy device deployment.

The output is shown in Figure 17. Only the output scores higher than 70 have been kept in order to highlight the most suitable areas.

The most suitable areas for onshore wave energy extraction are located in the southern part of the Atlantic coast as well as in French Brittany coasts, which are characterized by high incident wave energy.

Several studies (e.g. Hamm and Perronnard, 1997; Michard et al., 2013) have been undertaken in the framework of the EMACOP network (www.emacop.fr) in order to evaluate the nearshore/onshore wave energy potential over the French Atlantic area in different sites for devices deployment on existing coastal infrastructures (e.g. quay walls, breakwaters). The sites identified in those studies (e.g. Saint-Jean-De-Luz Bay, Bayonne, Esquibien) as being appropriate for wave energy extraction are in agreement with the most suitable areas found by the multi-criteria analysis tool developed in this study. This finding shows that the methodology developed in this study can be used, as a perspective, for nearshore and onshore site selection. However, several other parameters should be considered locally, such as wave parameters values at a higher resolution, the bathymetry and the slope of the area, the characteristics of the existing infrastructures and the socio-economical features (e.g. aquaculture, sailing) in order to validate the relevance of a particular site for a device deployment.

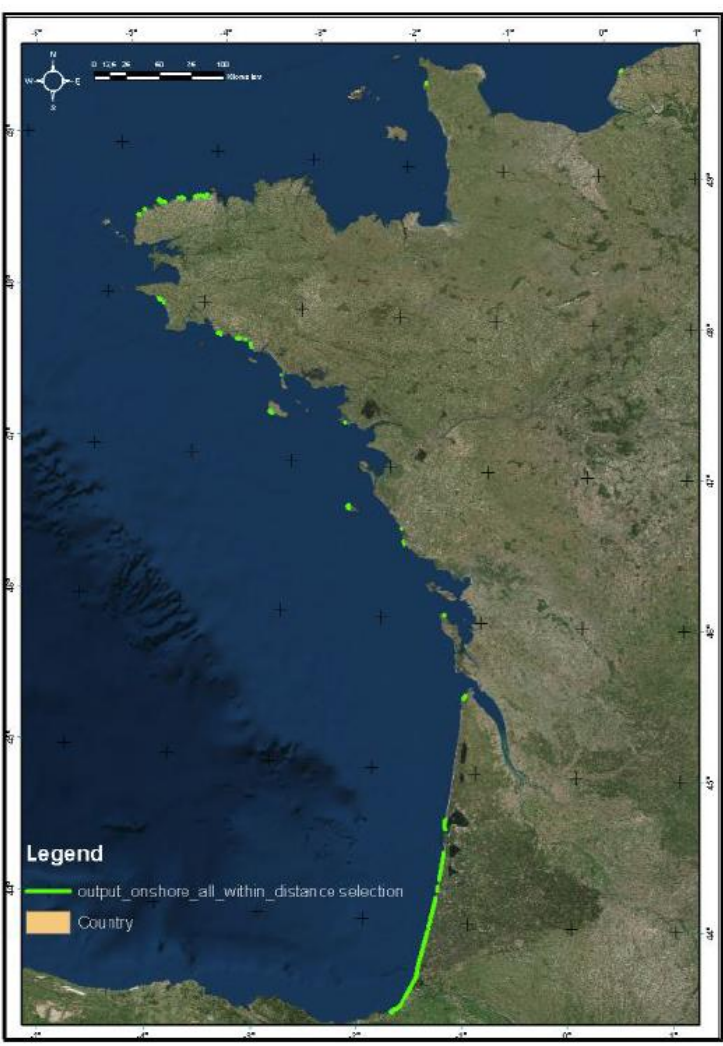

Figure 17. Most suitable areas for onshore wave energy devices deployment over the French Atlantic Area

\section{Conclusions}

The selection of a suitable site for wave energy projects is a complex task and much information is needed to conduct an informed evaluation. An accurate assessment of the available resource remains the most important factor, and this in itself requires considerable effort. In this study, a geospatial multi-criteria analysis method to identify the most suitable areas for wave energy devices deployment has been presented. It is applied to the French Atlantic area to distinguish the areas with higher wave power that meet the requirements of the multi-criteria selection methodology, using six parameters. Analysis shows that by including these variables, GIS is an effective approach to determine the best locations for energy extraction while minimizing environmental constraints.

A GIS was used to determine the geo-spatial suitability of the location for wave energy farms. This layer was generated based on point, line, polygon or matrix features. The first step of the methodology consists in establishing an analysis 
mask based on bathymetry, underwater cables and marine protected areas GIS data. Weighting factors (distances from the coastline, port areas and electrical substations, sea bottom characteristics and wave parameters) are created and extracted in the analysis mask (i.e. area of interest). Finally, the extracted data are reclassified to a common scale prior to the weighted overlay procedure, allowing the production of the final output (site suitability matrix). A suite of geo-processing models have been developed using ArcGIS $\odot$ ModelBuilder in order to make the system efficient and easily applicable. This paper has described the methodology and all the information that would be ideally required and hence should at least be taken into account. Nevertheless, the authors are conscious and wish to warn future users that in some cases, some data can be difficult to obtain, or even impossible.

The results showed that the most suitable places are located in the Atlantic coasts in the western part of France as well as in French Brittany, where the highest wave energy resources are located. Most suitable areas for wave energy devices deployment are those where the energy is high and the constraints are minimized.

The presented methodology is of general use. For that matter, both the reclassification and weighting procedures must be calibrated for specific wave energy system types and areas of interest. A major output of this technique is the evaluation of the economic potential of a certain system throughout a region or even the globe.

\section{Acknowledgements}

This work was funded by the European Regional Development Fund through the Atlantic Area Program.

\section{References}

[1] A. Nobre, M. Pacheco, R. Jorge, M. F. P. Lopes, L. M. C. Gato "Geo-spatial multi-criteria analysis for wave energy conversion system deployment". Renewable Energy, 34(1), pp 97-111, 2009.

[2] L. S. Yapa et all, "Is GIS appropriate technologyquest"; International Journal of Geographical Information Systems, 1991.

[3] A. Carrión, J. Espín Estrella, A. Aznar Dols, F. Zamorano Toro, M. Rodríguez, R. Ridao, "Environmental decision-support systems for evaluating the carrying capacity of land areas: Optimal site selection for gridconnected photovoltaic power plants". Renewable and Sustainable Energy Reviews, 12, (9), pp 2358-2380 2008.

[4] S. D. Brody, H. Grover, S. Bernhardt, Z. Tang, B. Whitaker, C. Spence, "Identifying potential conflict associated with oil and gas exploration in Texas state coastal waters: A multicriteria spatial analysis". Environmental Management, 38(4), 597-617 , 2006.

[5] M. Muselli, G. Notton, P. Poggi, A. Louche, "Computer-aided analysis of the integration of renewableenergy systems in remote areas using a geographicalinformation system". Applied Energy, 63(3), 141-160, 1999.

[6] A. S. Bayoumi, H. A. El-Gamal, "Research plan for harnessing wave energy in Egypt", IEEE 17th International Conference on Industrial Engineering and Engineering Management (IE\&EM), 2010.

[7] R. Prest, T. Daniell, B. Ostendorf, "Using GIS to evaluate the impact of exclusion zones on the connection cost of wave energy to the electricity grid". Energy Policy, 35(9), 4516-4528, 2007.

[8] N. Maslov, D. Brosset, C. Claramunt, J. F. Charpentier "A flexible decision-aid system for sites selection and technology options for a marine energy system", Renewable Power Generation Conference (RPG), 3rd, IET, 2014.

[9] H. S. Hansen, "GIS-based Multi-Criteria Analysis of Wind Farm Development". In Scandinavian Research Conference on Geographical Information Science, (pp. 7587), 2015.

[10] S. Carver, "Integrating multi-criteria evaluation with geographical information systems". International Journal of Geographical Information Science, 5(3), 321-339, 1991.

[11] M. Jakobsson, R. Macnab, N. Cherkis, H.-W. Schenke (2014), "The International Bathymetric Chart of the Arctic Ocean (IBCAO)". Research Publication RP-2, 1-13, 2014. 